\title{
Satellite-based analysis of thermosphere response to extreme solar flares
}

\author{
S. Krauss, M. Pfleger, and H. Lammer \\ Space Research Institute, Austrian Academy of Sciences, Schmiedlstr. 6, 8042 Graz, Austria \\ Correspondence to: S. Krauss (sandro.krauss@oeaw.ac.at)
}

Received: 27 March 2014 - Revised: 22 September 2014 - Accepted: 23 September 2014 - Published: 28 October 2014

\begin{abstract}
We present a refined algorithm to calculate pseudo solar indices, which enable the reproduction of a solar flare impact on the upper Earth's atmosphere for the empirical thermosphere model Jacchia-Bowman 2008. In a first step we compare the estimates of the new algorithm with those from a previous study by analysing an extreme X17.2 flare in 2003 using TIMED/SEE EUV observations. In a second step we adapt the method to use SOHO/SEM measurements within the algorithm and compare the findings with the previous results. Furthermore, the latter procedure is validated by means of GRACE density measurements during a X2.0 solar flare in November 2004. In each of the cases also a comparison with theoretical thermosphere models is performed, which shows a good agreement and suggests that the algorithm can support theoretical evolution studies in case no in situ density measurements during extreme solar events are available.
\end{abstract}

Keywords. Atmospheric composition and structure (evolution of the atmosphere; pressure, density, and temperature) solar physics, astrophysics, and astronomy (flares and mass ejections)

\section{Introduction}

The evolution of the Earth's atmosphere can only be understood if one considers that the radiation and particle environment of the Sun changed during its lifetime. According to recent studies (Tian et al., 2008; Lichtenegger et al., 2010; Lammer et al., 2011, 2013) related to the heating of the early Earth's upper atmosphere by extreme ultraviolet radiation (EUV), the young Sun could raise thermospheric temperatures up to several thousands of kelvin. For solar EUV fluxes larger than 5 times the present value, the thermosphere changes from a hydrostatic to a dynamically expanding nonhydrostatic regime (Tian et al., 2008). In such a regime the expanding upper thermosphere adiabatically cools but its exobase level can move beyond the location of present Earth's magnetopause distance. Thus, the magnetosphere is not able to protect the upper atmosphere from solar wind erosion. As shown by Lichtenegger et al. (2010) a $\mathrm{N}_{2}$-rich terrestrial atmosphere would have been lost within a few million years during the EUV active period of the young Sun about 4 Gyr ago. Since these studies are based mainly on theoretical methods, a validation through in situ observation is highly desirable. As discussed by Lammer et al. (2012) and shown by Krauss et al. (2012), low Earth orbiting satellites (LEOs) such as the Gravity Recovery and Climate Experiment (GRACE; Tapley et al., 2004) are suitable to study how the Earth's upper atmosphere responds to density enhancements related to the radiation and plasma environment of active solar events. Since GRACE is orbiting near the exobase level $(\sim 500 \mathrm{~km})$, its observations are predestined to be used as a diagnostic tool for retrieving thermospheric density and its variations during extreme solar events. Such events can be considered as proxies for periods in which the Sun was younger and more active (Krauss, 2013). However, due to the $90 \mathrm{~min}$ revolution period of GRACE it is quite common that the spacecraft can not observe the full impact of a solar flare occurring on the dayside of the Earth - in such cases an alternative solution would be desirable.

As we know from previous studies mentioned above, empirical thermosphere models (e.g. Jacchia-Bowman 2008, JB08, Bowman et al., 2008) are not constructed to reproduce the effects of short-term perturbations caused by solar flares due to the fact that these models use daily averaged solar indices (e.g. $F_{10.7}$ solar radio flux). Thus, the exobase density and exospheric temperature differ significantly from those 
obtained by theoretical thermosphere models (Kulikov et al., 2007; Tian et al., 2008). However, for the famous X17.2 solar flare on 28 October 2003, Krauss et al. (2012) have shown that it is possible to achieve similar results as Tian et al. (2008) by using estimated pseudo solar indices which reflect the peak intensity of the specific solar flare. Unfortunately, for this flare it was not possible to verify the pseudo solar index calculations by GRACE observations since the spacecraft was on the nightside and recorded only an aftermath of the flare impact.

The aim of this pursuing study is to validate the method for estimating pseudo solar indices presented by Krauss et al. (2012) by analysing a X2.0 solar flare in November 2004. For this specific event the period between the flare eruption and the first perturbations in the GRACE accelerometer measurements was rather small ( $<10 \mathrm{~min}$.). Therefore we can deduce that the spacecraft actually recorded the peak intensity of the flare and may serve as a benchmark for the neutral density. To begin with, Sect. 2 briefly introduces the data used in this study. Section 3 presents a revised version of the computation method for pseudo solar indices, which allows the usage of EUV observations from the missions Thermosphere Ionosphere Mesosphere Energetics and Dynamics (TIMED) and Solar and the Heliospheric Observatory (SOHO). In Sect. 4 the actual validation is performed by using density measurements from GRACE; furthermore a comparison between our estimates and the results from the theoretical models at different radiation levels is carried out. Finally, in Sect. 5, the conclusions of this study are summarised.

\section{Data}

Since this study makes use of observations from different satellite missions, the data products are introduced together with a brief description of the missions themselves. In order to get information about the condition of the Earth's upper atmosphere, we process accelerometer measurements ${ }^{1}$ from GRACE. The twin-satellite mission was launched in March 2002 and placed in a near-circular polar orbit at an initial altitude of $505 \mathrm{~km}$. Both spacecraft are orbiting approximately $220 \mathrm{~km}$ apart from each other with the main objective to detect temporal variations of the Earth's gravity field. For this purpose, the spacecraft are equipped with accelerometers, which allow the measurement of the non-gravitational forces. However, in our study we will use these observations to estimate neutral atmospheric densities along the trajectories of the satellites. For a detailed description on how such neutral densities can be calculated, we refer the reader to Krauss et al. (2012) and Krauss (2013).

In order to obtain knowledge about the radiation intensity during an extreme solar event, we use data from the missions TIMED and SOHO. The former mission is operated by NASA and was launched on 7 December 2001. The satellite

\footnotetext{
${ }^{1}$ http://isdc.gfz-potsdam.de/index.php
}

orbits Earth at an altitude of $\sim 625 \mathrm{~km}$ and is equipped with the Solar EUV Experiment (SEE). This instrument measures the irradiance of the solar soft X-ray, EUV and far ultraviolet radiation (26-194 nm) 12 to 15 times per day. During periods where TIMED observations are unavailable, we access data from the Solar Extreme ultraviolet Monitor $\left(\mathrm{SEM}^{2}\right.$ ) aboard the SOHO satellite, which operates in the Lagrange point $\mathrm{L}_{1}$ and also provides EUV measurements but in a smaller band pass (26-34 nm) with a temporal resolution of $15 \mathrm{~s}$.

\section{Methods}

The current algorithm to estimate pseudo solar indices $\left(I_{\mathrm{P}}\right)$ for the purpose of reproducing the effect of short-term events from solar flares is a refined version of that one presented by Krauss et al. (2012). Thus, we will initially re-analyse the X17.2 solar flare which occurred on 28 October 2003 and compare the estimates with those from Krauss et al. (2012).

\subsection{EUV measurements: TIMED/SEE}

In contrast to the previous version, where we only calculated a multiplication factor depending on the gradient of the EUV flux from TIMED/SEE and the solar index, the new formalism is based on an empirical relationship between solar indices and integrated EUV fluxes (Richards et al., 1994) and defined as

$I_{\mathrm{P}}=I_{0}\left(1+k\left[\frac{E_{\text {flare }}}{E_{0}}-1\right]\right)$.

Here $I_{0}$ denotes an average of each individual solar index $\left(F_{10.7}, S_{10.7}, M_{10.7}\right.$ and $\left.Y_{10.7}\right)$ used by the JB08 model and $E_{0}$ the mean of the integrated intensity flux over the EUV range 25-92 nm measured by TIMED/SEE. The peak EUV irradiance during the flare event is described by $E_{\text {flare }}$, and $k$ indicates a dimensionless scaling factor for each index, which can be written as

$k=\frac{I_{\max }-I_{0}}{E_{\max }-E_{0}} \cdot \frac{E_{0}}{I_{0}}$.

It should be noted that the ratios between the scaling factors $k$ remain unchanged even if the source of the intensity flux changes. $E_{\max }$ in Eq. (2) denotes the maximum value of the integrated intensity flux and $I_{\max }$ that from each solar index over a certain time span. In the case of the solar indices both maximum and average values are estimated between 1997 and 2008 in order to comprise one solar cycle. Since the TIMED/SEE mission was launched in 2002, it has been impossible to choose the same period to calculate $E_{0}$ and $E_{\max }$. Therefore, we have decided to evaluate the period from 2002 to 2008 (Fig. 1, left). The resulting numerical values for the input parameters are summarised in Table 1,

\footnotetext{
${ }^{2}$ http://www.usc.edu/dept/space_science/semdatafolder/ semdownload.htm
} 
Table 1. Average and maximum values of the different solar indices and the integrated intensity flux measurements [25-92 $\mathrm{nm}$ ] by TIMED/SEE.

\begin{tabular}{lll}
\hline Input parameter & Numerical value & Source \\
\hline$E_{0}$ & $0.0017753\left[\mathrm{~W} \mathrm{~m}^{-2}\right]$ & SEE EGS L2 \\
$E_{\max }$ & $0.0031971\left[\mathrm{~W} \mathrm{~m}^{-2}\right]$ & SEE EGS L2 \\
$E_{\text {flare }}$ & $0.0046337\left[\mathrm{~W} \mathrm{~m}^{-2}\right]$ & SEE EGS L2A \\
$F_{0} / F_{\max }$ & $120.2 / 226.7[\mathrm{sfu}]$ & JB08 database \\
$S_{0} / S_{\max }$ & $111.0 / 213.3[\mathrm{sfu}]$ & JB08 database \\
$M_{0} / M_{\max }$ & $118.8 / 214.4[\mathrm{sfu}]$ & JB08 database \\
$Y_{0} / Y_{\max }$ & $117.9 / 180.2[\mathrm{sfu}]$ & JB08 database \\
\hline
\end{tabular}

${ }^{a}$ http://lasp.colorado.edu/see/; b http://sol.spacenvironment.net/ jb2008/
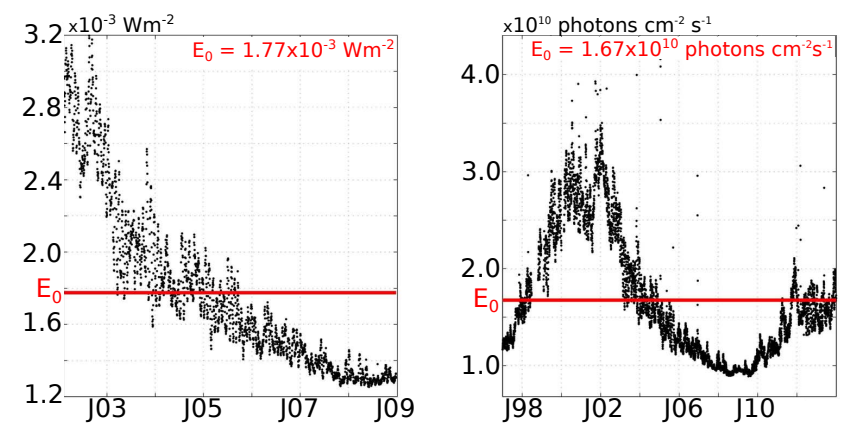

Figure 1. Integrated intensity flux of the daily averages by TIMED/SEE (25-92 nm, left) and SOHO/SEM (26-34 nm, right). Data from the latter mission will be used in Sect. 3.2.

together with the peak intensity of the EUV radiation during the X17.2 flare.

Based on these values it is possible to evaluate Eqs. (1) and (2) to obtain the desired pseudo solar indices which reflect the peak intensity of the X17.2 solar flare. Table 2 presents the pseudo solar indices and the corresponding scaling factors based on EUV observations from TIMED/SEE together with the estimates from Krauss et al. (2012).

\subsection{EUV measurements: SOHO/SEM}

Since TIMED/SEE provides EUV observations only every $90 \mathrm{~min}$, it is very rare that the satellite observes the peak intensity of a solar flare. For this reason, we adapted the algorithm in such a way that the usage of continuous EUV measurements from SOHO/SEM is possible. In doing so, we analysed almost the entire mission duration (Fig. 1, right) to obtain a mean integration flux of $1.670 \times$ $10^{10}$ photons $\mathrm{cm}^{-2} \mathrm{~s}^{-1}$ at $1 \mathrm{AU}$. Besides the continuous observations from SOHO/SEM, another advantage is that we can use the same formalism to calculate the $S_{10.7}$ index (cf. Eq. 3; Tobiska et al., 2008) as used for the JB08 model,

$S_{10.7}=-12.01+141.23 \times\left(\mathrm{ph}_{26-34} / 1.9955 \times 10^{10}\right)$.
Table 2. Estimated pseudo solar indices to reproduce the peak EUV radiation during the X17.2 solar flare on 28 October 2003 using TIMED/SEE observations.

\begin{tabular}{lllr}
\hline $\begin{array}{l}\text { Pseudo } \\
\text { indices }\end{array}$ & $\begin{array}{l}\text { Krauss } \\
\text { et al }(2012)\end{array}$ & $\begin{array}{l}\text { Present study } \\
\text { TIMED/SEE }\end{array}$ & $\begin{array}{l}\text { Corresponding } \\
\text { scale factor }(k)\end{array}$ \\
\hline$F_{\text {flare }}$ & $334.4[\mathrm{sfu}]$ & $334.3[\mathrm{sfu}]$ & 1.106 \\
$S_{\text {flare }}$ & $307.1[\mathrm{sfu}]$ & $316.7[\mathrm{sfu}]$ & 1.151 \\
$M_{\text {flare }}$ & $319.6[\mathrm{sfu}]$ & $311.0[\mathrm{sfu}]$ & 1.005 \\
$Y_{\text {flare }}$ & $256.3[\mathrm{sfu}]$ & $243.1[\mathrm{sfu}]$ & 0.660 \\
\hline
\end{tabular}

But instead of using daily averaged values we insert the highrate measurements from SOHO/SEM $\left(\mathrm{ph}_{26-34}\right)$ during the flare event. Thus, the resulting $S_{10.7}$ index should be comparable with our previously estimated pseudo index $S_{\text {flare }}$ in Table 2.

During the peak intensity of the X17.2 flare at 11:10 UT, the SOHO/SEM measured a flux of $4.593 \times$ $10^{10}$ photons $\mathrm{cm}^{-2} \mathrm{~s}^{-1}$ at $1 \mathrm{AU}$. By inserting this value into Eq. (3), we obtain a high-rate $S_{10.7}$ index of the order of $313.06 \mathrm{sfu}$. A subsequent evaluation of Eq. (1) yields a scaling factor for the $S$ index of $k=1.04$. Since the ratio between the $k$ factors is already known, we can also determine the remaining pseudo solar indices using Eq. (1). Table 3 lists the outcomes of this procedure.

Under the prerequisite that the Sun had a constant radiation in the order of the X17.2 flare, we can re-evaluated the JB08 model by replacing both input parameter (daily and 81day average indices) with the pseudo solar indices of Table 2 and 3. The resulting mass densities and the exobase temperatures are listed in Table 4 together with the values published in Krauss et al. (2012).

Compared to the outcome by Krauss et al. (2012), the new density estimates are slightly higher and closer to the estimates by Tian et al. (2008). Since the initial method for estimating pseudo solar indices contained some simplifications, especially for the ratio between the EUV radiation and the JB08 indices which was based only on 20 selected points, the current results ought to be more reliable and are preferred. Furthermore, we see a good accordance between the estimates using TIMED/SEE and SOHO/SEM; thus it appears to be justified also to use SOHO/SEM measurements to estimate pseudo solar indices.

\section{Validation}

In order to use GRACE as a validation tool, it is mandatory to find a solar flare where the spacecraft recorded the disturbances within a few minutes - equivalent to the maximum impact of the flare. However, during the search for such an appropriate solar flare some difficulties arose. First of all, large X-class flares mostly occur during high solar activity, but since our GRACE analysis starts in 2003 only the first 
Table 3. Estimated pseudo solar indices and corresponding scaling factors for the X17.2 solar flare in 2003 using SOHO/SEM observations.

\begin{tabular}{llr}
\hline $\begin{array}{l}\text { Pseudo } \\
\text { indices }\end{array}$ & $\begin{array}{l}\text { Present study } \\
\text { SOHO/SEM }\end{array}$ & $\begin{array}{r}\text { Corresponding } \\
\text { scale factor }(k)\end{array}$ \\
\hline$F_{\text {flare }}$ & $330.4[\mathrm{sfu}]$ & 0.999 \\
$S_{\text {flare }}$ & $313.1[\mathrm{sfu}]$ & 1.040 \\
$M_{\text {flare }}$ & $307.6[\mathrm{sfu}]$ & 0.908 \\
$Y_{\text {flare }}$ & $240.9[\mathrm{sfu}]$ & 0.596 \\
\hline
\end{tabular}

Table 4. Estimates for exobased densities and exosphere temperatures from various source, including the algorithm described in Krauss et al. (2012), Tian et al. (2008), Kulikov et al. (2007) and the results from the present study using TIMED/SEE and SOHO/SEM data.

\begin{tabular}{lcr}
\hline Source & $\begin{array}{c}\text { Exobase } \\
\text { density }\left[\mathrm{kg} \mathrm{m}^{-3}\right]\end{array}$ & $\begin{array}{r}\text { Exosphere } \\
\text { temperature }[\mathrm{K}]\end{array}$ \\
\hline Present study (TIMED) & $2.12 \times 10^{-11}$ & 1988 \\
Present study (SOHO) & $2.02 \times 10^{-11}$ & 1980 \\
Krauss et al. (2012) & $1.70 \times 10^{-11}$ & 1950 \\
Tian et al. (2008) $\left(2.5 E_{0}\right)$ & $2.30 \times 10^{-11}$ & $\sim 1800$ \\
Kulikov et al. (2007) $\left(3.0 E_{0}\right)$ & $1.24 \times 10^{-11}$ & $\sim 2400$ \\
\hline
\end{tabular}

few years are of interest. During solar minima the flares are much smaller and therefore also their impact on the spacecraft. Another limiting factor is that the requested flare must not be superimposed by the impact of an interplanetary coronal mass ejection (ICME), as it was the case with the X10 flare on 29 October 2003. In such a case a separation between the effect of the ICME and the flare is impossible. Last but not least, a suitable location of the GRACE satellite, which means that the spacecraft is on the dayside near the equator during the eruption, is not given at all times.

A solar flare fulfilling most requirements happened on 7 November 2004. During this specific event the GRACE satellites recorded the first impact at $\sim 16: 15 \mathrm{UT}-9 \mathrm{~min}$ after the peak intensity of the flare. Thus, the measured densities can be considered as a benchmark for the theoretical models and the JB08 model when applying pseudo solar indices.

In Fig. 2 an illustration of the atmospheric density variations at the beginning of November 2004 is shown. Eyecatching are the large disturbances on 8 November (region 2) which can be attributed to a geomagnetic storm triggered by an ICME on 4 November (Richardson and Cane, 2010). These kinds of disturbances are currently under investigation and thus not the subject of the present study. Our attention is focussed on the sudden increase due to the X2.0 flare radiation (Fig. 2, region 1).

As expected, the JB08 model is only able to reproduce the impact of the ICME and can not model the short-term perturbation of the solar flare due to the usage of averaged

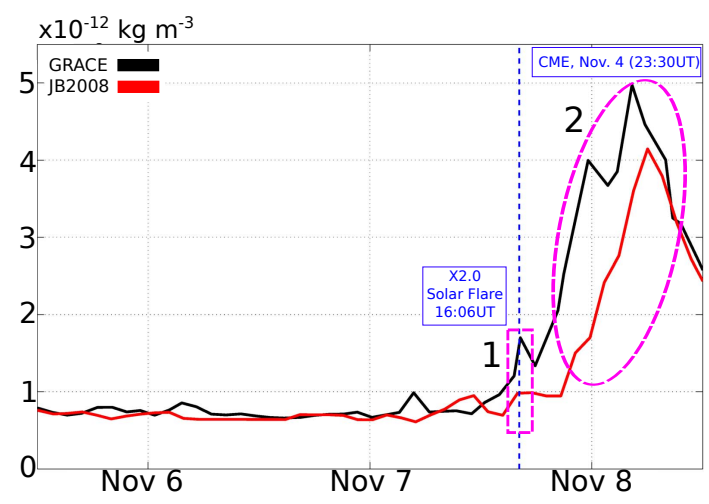

Figure 2. Neutral densities from GRACE measurements (black) and the empirical thermosphere model JB08 (red) using average daily indices in November 2004. Region 1 shows the sudden increase due to the X2.0 solar flare, whereas region 2 indicates the impact of an ICME a few hours later.

Table 5. Estimated pseudo indices and the corresponding scale factor $k$ for the X2.0 solar flare on 7 November 2004 using SOHO/SEM observations.

\begin{tabular}{llr}
\hline $\begin{array}{l}\text { Pseudo } \\
\text { indices }\end{array}$ & $\begin{array}{l}\text { Present study } \\
\text { SOHO/SEM }\end{array}$ & $\begin{array}{r}\text { Corresponding } \\
\text { scaling factor } k\end{array}$ \\
\hline$F_{\text {flare }}$ & $140.0[\mathrm{sfu}]$ & 0.816 \\
$S_{\text {flare }}$ & $130.0[\mathrm{sfu}]$ & 0.849 \\
$M_{\text {flare }}$ & $136.6[\mathrm{sfu}]$ & 0.741 \\
$Y_{\text {flare }}$ & $129.5[\mathrm{sfu}]$ & 0.487 \\
\hline
\end{tabular}

solar indices. Thus, the estimated neutral densities are significantly lower $\left(1.00 \times 10^{-12} \mathrm{~kg} \mathrm{~m}^{-3}\right)$ than those measured by GRACE $\left(1.83 \times 10^{-12} \mathrm{~kg} \mathrm{~m}^{-3}\right)$. However, based on the above described algorithm it should be possible to obtain similar results from the JB08 model if we use the pseudo solar indices.

Since there are no TIMED/SEE observations available during the X2.0 solar flare on 7 November 2004, we will use EUV observations from SOHO/SEM. During this specific flare the satellite measured an integrated flux of $2.006 \times$ $10^{10}$ photons $\mathrm{cm}^{-2} \mathrm{~s}^{-1}$ at $1 \mathrm{AU}$. Based on this observation we can apply the above-described procedure to estimate pseudo solar indices $F_{10.7}, M_{10.7}$ and $Y_{10.7}$ (Table 5).

By using these indices as input parameters for the JB08 model, we obtain a neutral density of $1.70 \times 10^{-12} \mathrm{~kg} \mathrm{~m}^{-3}$, which is in good agreement with our previously defined benchmark - the GRACE measurement $(1.83 \times$ $10^{-12} \mathrm{~kg} \mathrm{~m}^{-3}$ ). Thus, we conclude that the validation of our algorithm to calculate pseudo solar indices was successful.

Regarding the exobase temperature, which is also estimated by the JB08 model, the re-evaluation yields a value of $\sim 1170 \mathrm{~K}$ for the X2.0 solar flare $\left(1.2 \times E_{0}\right)$. This result is in line with the temperatures from theoretical models by Tian et al. (2008) of $\sim 1050 \mathrm{~K}$ and Kulikov et al. (2007) of 1014 K (Fig. 3). 


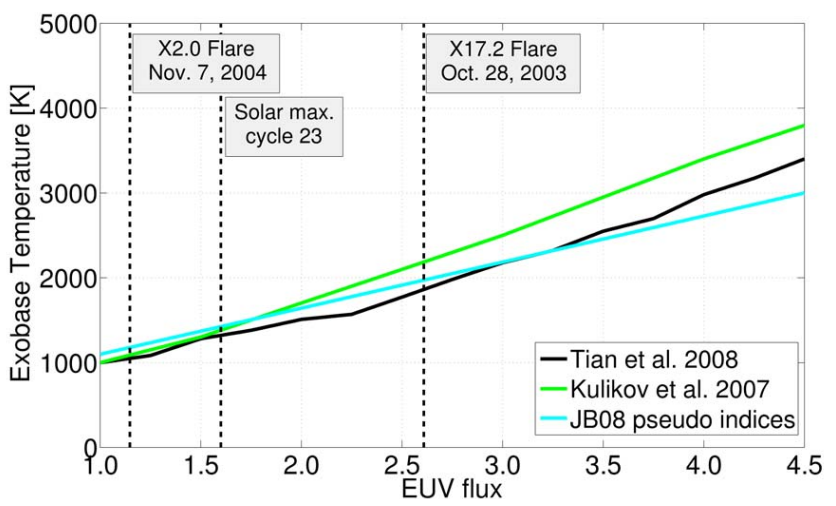

Figure 3. Exobase temperatures obtained from the empirical thermosphere model JB08 using the pseudo solar indices and the theoretical models of Kulikov et al. (2007) and Tian et al. (2008) under different solar EUV conditions.

\section{Conclusions}

In summary it can be stated that the algorithm for the calculation of pseudo solar indices seems to be stable and that the usage of these indices within the JB08 model permits a meaningful comparison between empirical and theoretical upper atmosphere models. Furthermore, it has been shown that the method provides almost identical results for different types of EUV measurements (TIMED/SEE, SOHO/SEM). However, for future research it would be desirable to analyse the peak intensity of a solar flare by all three satellite missions (GRACE, SOHO, TIMED) - so far this scenario has never been given. Regarding the observations by GRACE it has been shown that if the satellites are in a suitable orbit position the observations can be used to directly extract information concerning the influence of a solar flare event on the Earth's upper atmosphere in order to support the theoretical models.

Topical Editor C. Jacobi thanks two anonymous referees for their help in evaluating this paper.

\section{References}

Bowman, B. R., Tobiska, W. K., Marcos, F. A., Huang, C. Y., Lin, C. S., and Burke, W. J.: A New Empirical Thermospheric Density Model JB2008 Using New Solar and Geomagnetic Indices, AIAA/AAS Astrodynamics Specialist Conference, AIAA 20086438, 2008.

Krauss, S.: Response of the Earth's thermosphere during extreme solar events. A contribution of satellite observations to atmospheric evolution studies, $\mathrm{PhD}$. thesis, Graz University of Technology, 2013.

Krauss, S., Fichtinger, B., Lammer, H., Hausleitner, W., Kulikov, Yu. N., Ribas, I., Shematovich, V. I., Bisikalo, D., Lichtenegger, H. I. M., Zaqarashvili, T. V., Khodachenko, M. L., and Hanslmeier, A.: Solar flares as proxy for the young Sun: satellite observed thermosphere response to an X17.2 flare of Earth's upper atmosphere, Ann. Geophys., 30, 1129-1141, doi:10.5194/angeo-30-1129-2012, 2012.

Kulikov, Y. N., Lammer, H., Lichtenegger, H. I. M., Penz, T., Breuer, D., Spohn, T., Lundin, R., and Biernat, H. K.: A Comparative Study of the Influence of the Active Young Sun on the Early Atmospheres of Earth, Venus and Mars, Space Sci. Rev., 129, 207-243, 2007.

Lammer, H., Güdel, M., Kulikov, Yu. N., Ribas, I., Zaqarashvili, T. V., Khodachenko, M. L., Kislyakova, K. G., Gröller, H., Odert, P., Leitzinger, M., Fichtinger, B., Krauss, S., Hausleitner, W., Holmström, M., Sanz-Forcada, J., Lichtenegger, H. I. M., Hanslmeier, A., Shematovich, V. I., Bisikalo, D., Rauer, H., and Fridlund, M.: Variability of solar/stellar activity and magentic field and its influence on planetary atmosphere evolution, Earth Planets Space, 64, 179-199, doi:10.5047/eps.2011.04.002, 2012.

Lammer, H., Kislyakova, K. G., Odert, P., Leitzinger, M., Schwarz, R., Lohinger, E. P., Kulikov, Yu. N., Khodachenko, M. L., Güdel, M., and Hanslmeier, A.: Pathways to Earth-Like Atmospheres. Extreme Ultraviolet (EUV)-Powered Escape of Hydrogen-Rich Protoatmospheres, Orig. Life Evol. Biosp., 41, 503-522, doi:10.1007/s11084-012-9264-7, 2012.

Lammer, H., Kislyakova, K. G., Güdel, M., Holmström, M., Erkaev, N. V., Odert, P., and Khodachenko, M. L.: Stability of Earth-Like $\mathrm{N}_{2}$ Atmospheres: Implications for Habitability, The Early Evolution of the Atmosphere of Terrestrial Planets, Astrophysics and Space Science Proceedings 35, doi:10.1007/978-1-4614-51914_4, Springer Science+Business Media New York, 2013.

Lichtenegger, H. I. M., Lammer, H., Grießmeier, J. N., Kulikov, Y. N., von Paris, P., Hausleitner, W., Krauss, S., and Rauer, H.: Aeronomical evidence for higher $\mathrm{CO}_{2}$ levels during Earth's Hadean epoch, Icarus, 210, 1-7, doi:10.1016/j.icarus.2010.06.042, 2010.

Richards, P. G., Fennelly, J. A., and Torr, D. G.: EUVAC: A solar EUV flux model for aeronomic calculations, J. Geophys. Res., 99, 8981-8992, 1994.

Richardson, I. G. and Cane, H. V.: Interplanetary Coronal Mass Ejections During Solar Cycle 23, AIP Conference Proceedings 1216, 683, doi:10.1063/1.3395959, 2010.

Tapley, B. D., Bettadpur, S., Watkins, M., and Reigber, C.: The Gravity Recovery and Climate Experiment: Mission Overview and Early Results, Geophys. Res. Lett., 31, L09607, doi:10.1029/2004GL019920, 2004.

Tian, F., Kasting, J. F., Liu, H. L., and Roble, R. G.: Hydrodynamic planetary thermosphere model: 1 . Response of the Earth's thermosphere to extreme solar EUV conditions and the significance of adiabatic cooling, J. Geophys. Res.-Planets, 113, 05008, doi:10.1029/2007JE002946, 2008.

Tobiska, W. K., Bowman, B. R., and Bouwer, S. D.: Solar and geomagnetic indices for the JB2008 thermosphere density model, COSPAR CIRA Draft, Chapter 4, available at: http://sol. spacenvironment.net/ jb2008/publications.html, 2008. 